\title{
The Theory of Soft Ditopological Spaces
}

\author{
Güzide Şenel \\ Department of Mathematics, Faculty of Arts and Sciences, \\ Amasya University, 05100 Amasya, Turkey,
}

\begin{abstract}
In this paper, by defining soft ditopological spaces, a new approach to the soft topology via soft set theory is made, with defining two structures on a soft set - a soft topology and a soft subspace topology. Moreover, separation axioms in soft ditopological spaces are characterized and the relations between soft topological and soft ditopological structures are investigated.
\end{abstract}

\section{General Terms}

2010 AMS Classification: 54A10, 28C15, 54J05, 54A05, 03G25, 20D05, $03 H 05$.

\section{Keywords}

Soft sets, soft open set, soft topology, soft topological subspace, soft ditopology.

\section{INTRODUCTION}

Since the exordium of soft sets was done by Molodtsov [10] in 1999, soft set theory has been regarded as a general mathematical instrument for dealing with uncertainties. After this invention, many related concepts with the theory and the application of soft sets have also undergone tremendous studies.

The theory of soft set has a balanced coverage of both theory and practice. In theoretical, Maji et al. [9] defined the operations based on the analysis of several operations on soft sets. By using this operations, the theory of soft topological space defined by Shabir and Naz [12] over an initial universe. Then, Aygünoglu and Aygün [2] introduced soft product topology and defined soft compactness. Also, soft set theory has a large scale on applications as in the following studies:[7, 8]

The theory of soft topology which is the mathematical formulation of approximate reasoning has become an important area of research in recent years. Because of a specific topological structure on a soft set is not sufficient in complicated applications, the discovery of an additional topological structure on the soft set needs to arise. To confirm this idea a soft bitopology over an initial universe was introduced in [6] by Ittanagi. In 2016, Dizman et al. [5] defined the concept of soft a soft ditopological space as the soft generalization of the concept of a ditopological space.

To make a specific work, this article is divided into three sections. The first section is introductory and it provides motivation for the development of the theory of ditopological spaces, describes its structure and outlines its potential for soft topological applications.
The second section is an overview of the basic concepts of soft set, soft point, soft topology and soft subspace topology necessary for characterization of the structure of soft ditopological spaces.

The main section of the manuscript is third which has two subsections. The first subsection extends the theory of soft topology to obtain an additional soft topological structure on the same soft set and introduce soft ditopological space, called soft ditopology that will be abbreviated by SDT. The concept of soft ditopological (SDT) space on a soft set with two structures on it is being introduced - a soft topology and a soft subspace topology. The first one is used to describe soft openness properties of a soft topological space while the second one deals with its sub - soft openness properties. The differences this work from [5] is, this structure enables to study with all soft open sets can be obtained on a soft set by the second structure named soft subspace toplogy. Definitions of soft open set, soft closed set, soft closure, soft interior and soft neighborhood in a soft ditopological space are given. Among the main properties of them, some important relations between soft interior and soft closure operations have been investigated. The comparison of soft ditopological spaces is defined. A constructive proof of every soft ditopological space of a soft set is a soft topological space over the soft set and a soft topological space is not need to be soft ditopological space is defined. Theorems characterizing behaviour of SDT, including deduction,contradiction and others are proved. The second subsection introduces separation axioms for a soft ditopological space $\left(f, \tilde{\tau}, \tilde{\tau}_{g}\right)$ by requesting corresponding separation properties for its soft topology $\tilde{\tau}$ and soft subspace topology $\tilde{\tau}_{g}$. A special attention has been paid to the definition of soft Hausdorff space in a soft ditopological space. Besides this, fundamentals of separation axioms that are substantiated by suitable examples in most of the occasions are also outlined. In this study, some of the new results and consequences, which could be useful from the point of view of soft set theory are presented.

\section{PRELIMINARY}

In this section, some basic notions relevant to soft set, soft point, soft topological space and soft topological subspace which may be found in [2, 4, 10, 11] are recalled for further details.

Note that throughout this paper, $U$ refers to an initial universe, $E$ is a set of parameters and $P(U)$ is the power set of $U$.

DEFINITION 1. 44 10 A soft set $f$ on the universe $U$ is is a set defined by

$$
f: E \rightarrow \mathcal{P}(U) \text { such that } f(e)=\emptyset \text { if } e \in E \backslash A \text { then, } f=f_{A}
$$


Here $f$ is also called an approximate function. A soft set over $U$ can be represented by the set of ordered pairs

$$
f=\{(e, f(e)): e \in E\}
$$

We will identify any soft set $f$ with the function $f(e)$ and and we shall use that concept as interchangeable. Soft sets are denoted by the letters $f, g, h, \ldots$ and the corresponding functions by $f(e), g(e)$, $h(e), \ldots$ Throughout this paper, the set of all soft sets over $U$ will be denoted by $S$. From now on, or all undefined concepts about soft sets, we refer to: [4].

Definition 2. [4] Let $f \in S$. Then,

If $f(e)=\emptyset$ for all $e \in E$, then $f$ is called an empty set, denoted by $\Phi$.

If $f(e)=U$ for all $e \in E$, then $f$ is called universal soft set, denoted by $\tilde{E}$.

Definition 3. [4] Let $f, g \in S$. Then,

$f$ is a soft subset of $g$, denoted by $f \widetilde{\subseteq} g$, if $f \subseteq g$ for all $e \in E$. $f$ and $g$ are soft equal, denoted by $f=g$, if and only if $f(e)=g(e)$ for all $e \in E$.

Definition 4. 4] $f, g \in S$. Then, the intersection of $f$ and $g$, denoted $f \widetilde{\cap} g$, is defined by

$$
(f \widetilde{\cap} g)(e)=f(e) \cap g(e) \text { for all } e \in E
$$

and the union of $f$ and $g$, denoted $f \widetilde{\cup} g$, is defined by

$$
(f \widetilde{\cup} g)(e)=f(e) \cup g(e) \text { for all } e \in E
$$

Definition 5. [4] $f \in S$. Then, the soft complement of $f$, denoted $\widetilde{f^{c}}$, is defined by

$$
\widetilde{f^{c}}(e)=U \backslash f(e), \text { for all } e \in E
$$

Definition 6. [4] Let $f \in S$. The power soft set of $f$ is defined by

$$
\mathcal{P}(f)=\left\{f_{i} \tilde{\subseteq} f: i \in I\right\}
$$

and its cardinality is defined by

$$
|\mathcal{P}(f)|=2^{\sum_{e \in E}|f(e)|}
$$

where $|f(e)|$ is the cardinality of $f(e)$.

EXAmple 1. Let $U=\left\{u_{1}, u_{2}, u_{3}\right\}$ and $E=\left\{e_{1}, e_{2}\right\} . f \in S$ and

$$
f=\left\{\left(e_{1},\left\{u_{1}, u_{2}\right\}\right),\left(e_{2},\left\{u_{2}, u_{3}\right\}\right)\right\}
$$

Then,

$$
\begin{aligned}
f_{1} & =\left\{\left(e_{1},\left\{u_{1}\right\}\right)\right\}, \\
f_{2} & =\left\{\left(e_{1},\left\{u_{2}\right\}\right)\right\}, \\
f_{3} & =\left\{\left(e_{1},\left\{u_{1}, u_{2}\right\}\right)\right\}, \\
f_{4} & =\left\{\left(e_{2},\left\{u_{2}\right\}\right)\right\}, \\
f_{5} & =\left\{\left(e_{2},\left\{u_{3}\right\}\right)\right\}, \\
f_{6} & =\left\{\left(e_{2},\left\{u_{2}, u_{3}\right\}\right)\right\}, \\
f_{7} & =\left\{\left(e_{1},\left\{u_{1}\right\}\right),\left(e_{2},\left\{u_{2}\right\}\right)\right\}, \\
f_{8} & =\left\{\left(e_{1},\left\{u_{1}\right\}\right),\left(e_{2},\left\{u_{3}\right\}\right)\right\}, \\
f_{9} & =\left\{\left(e_{1},\left\{u_{1}\right\}\right),\left(e_{2},\left\{u_{2}, u_{3}\right\}\right)\right\}, \\
f_{10} & =\left\{\left(e_{1},\left\{u_{2}\right\}\right),\left(e_{2},\left\{u_{2}\right\}\right)\right\}, \\
f_{11} & =\left\{\left(e_{1},\left\{u_{2}\right\}\right),\left(e_{2},\left\{u_{3}\right\}\right)\right\}, \\
f_{12} & =\left\{\left(e_{1},\left\{u_{2}\right\}\right),\left(e_{2},\left\{u_{2}, u_{3}\right\}\right)\right\}, \\
f_{13} & =\left\{\left(e_{1},\left\{u_{1}, u_{2}\right\}\right),\left(e_{2},\left\{u_{2}\right\}\right)\right\}, \\
f_{14} & =\left\{\left(e_{1},\left\{u_{1}, u_{2}\right\}\right),\left(e_{2},\left\{u_{3}\right\}\right)\right\}, \\
f_{15} & =f \\
f_{16} & =\Phi
\end{aligned}
$$

are all soft subsets of $f$. So $|\tilde{P}(f)|=2^{4}=16$.

DEFINITION 7. [?] The soft set $f$ is called a soft point in $S$, if for the parameter $e_{i} \in E$ such that $f\left(e_{i}\right) \neq \emptyset$ and $f\left(e_{j}\right)=\emptyset$, for all $e_{j} \in E \backslash\left\{e_{i}\right\}$ is denoted by $\left(e_{i_{f}}\right)_{j}$ for all $i, j \in N^{+}$.

Note that the set of all soft points of $f$ will be denoted by $S P(f)$.

EXAMPLE 2. [?] Let $U=\left\{u_{1}, u_{2}, u_{3}, u_{4}, u_{5}\right\}$ and $E=$ $\left\{e_{1}, e_{2}, e_{3}\right\} . f \in S$ and

$$
f=\left\{\left(e_{1},\left\{u_{1}, u_{3}\right\}\right),\left(e_{2},\left\{u_{2}, u_{3}\right\}\right),\left(e_{3},\left\{u_{1}, u_{2}, u_{3}\right\}\right)\right\}
$$

Then the soft points for the parameter $e_{1}$ are;

$$
\begin{aligned}
& \left(e_{1_{f}}\right)_{1}=\left(e_{1},\left\{u_{1}\right\}\right) \\
& \left(e_{1_{f}}\right)_{2}=\left(e_{1},\left\{u_{3}\right\}\right) \\
& \left(e_{1_{f}}\right)_{3}=\left(e_{1},\left\{u_{1}, u_{3}\right\}\right)
\end{aligned}
$$

one of them can be chosen as soft point.

For the the parameter $e_{2}$ one of three occasions can be chosen as soft point likewise;

$$
\begin{aligned}
& \left(e_{2_{f}}\right)_{1}=\left(e_{2},\left\{u_{2}\right\}\right) \\
& \left(e_{2_{f}}\right)_{2}=\left(e_{2},\left\{u_{3}\right\}\right) \\
& \left(e_{2_{f}}\right)_{3}=\left(e_{2},\left\{u_{2}, u_{3}\right\}\right)
\end{aligned}
$$

The soft points for the parameter $e_{3}$ are;

$$
\begin{aligned}
& \left(e_{3_{f}}\right)_{1}=\left(e_{3},\left\{u_{1}\right\}\right) \\
& \left(e_{3_{f}}\right)_{2}=\left(e_{3},\left\{u_{2}\right\}\right) \\
& \left(e_{3_{f}}\right)_{3}=\left(e_{3},\left\{u_{3}\right\}\right) \\
& \left(e_{3_{f}}\right)_{4}=\left(e_{3},\left\{u_{1}, u_{2}\right\}\right) \\
& \left(e_{3_{f}}\right)_{5}=\left(e_{3},\left\{u_{1}, u_{3}\right\}\right) \\
& \left(e_{3_{f}}\right)_{6}=\left(e_{3},\left\{u_{2}, u_{3}\right\}\right) \\
& \left(e_{3_{f}}\right)_{7}=\left(e_{3},\left\{u_{1}, u_{2}, u_{3}\right\}\right)
\end{aligned}
$$

Definition 8. [2] Let $f \in S$. A soft topology on $f$, denoted by $\tilde{\tau}$, is a collection of soft subsets of $f$ having following properties:

i. $f, \Phi \in \tilde{\tau}$,

ii. $\left\{g_{i}\right\}_{i \in I} \subseteq \tilde{\tau} \Rightarrow \tilde{U}_{i \in I} g_{i} \in \tilde{\tau}$,

iii. $\left\{g_{i}\right\}_{i=1}^{n} \subseteq \tilde{\tau} \Rightarrow \tilde{\bigcap}_{i=1}^{n} g_{i} \in \tilde{\tau}$.

The pair $(f, \tilde{\tau})$ is called a soft topological space.

EXAmple 3. Refer exam 1. $\tilde{\tau}^{1}=\tilde{\mathcal{P}}(f), \tilde{\tau}^{0}=\{\Phi, f\}$ and $\tilde{\tau}=\left\{\Phi, f, f_{2}, f_{11}, f_{13}\right\}$ are soft topologies on $f$.

DEFINITION 9. [2] Let $(f, \tilde{\tau})$ be a soft topological space. Then, every element of $\tilde{\tau}$ is called soft open set. Clearly, $\Phi$ and $f$ are soft open sets.

THEOREM 10. [11] If $F$ is a collection of soft closed sets in a soft topological space $(f, \tilde{\tau})$, then

$i$. Universal soft set $\tilde{E}$ is soft closed.

ii. Any intersection of members of $F$ belongs to $F$. iii. Any finite union of members of $F$ belongs to $F$.

Since $\tilde{E}^{\tilde{c}}=\Phi \in \tilde{\tau}, \tilde{E}$ is soft closed. But, $\Phi$ and $f$ need not to be soft closed. The following example shows that: 
EXAMPLE 4. Consider the topology $\tilde{\tau}=\left\{\Phi, f, f_{2}, f_{11}, f_{13}\right\}$ is defined in exam 1 Here, $f$ and $\Phi$ are not soft closed sets because

$f^{\tilde{c}}=\left\{\left(e_{1},\left\{u_{3}\right\}\right),\left(e_{2},\left\{u_{1}\right\}\right)\right\} \notin \tilde{\tau}$ and $\Phi^{\tilde{c}}=\tilde{E} \notin \tilde{\tau}$.

THEOREM 11. [11] Let $(f, \tilde{\tau})$ be a soft topological space and $g \subseteq \tilde{\subseteq}$. Then, the collection

$$
\tilde{\tau}_{g}=\{h \tilde{\cap} g: h \in \tilde{\tau}\}
$$

is a soft topology on $g$ and the pair $\left(g, \tilde{\tau}_{g}\right)$ is a soft topological space.

DEFINITION 12. [11] Let $(f, \tilde{\tau})$ be a soft topological space and $g \subseteq \tilde{\subseteq}$. Then, the collection

$$
\tilde{\tau}_{g}=\{h \tilde{\cap} g: h \in \tilde{\tau}\}
$$

is called a soft subspace topology on $g$ and $\left(g, \tilde{\tau}_{g}\right)$ is called a soft topological subspace of $(f, \tilde{\tau})$.

THEOREM 13. [11] Let $\left(g, \tilde{\tau}_{g}\right)$ be a soft subspace of a soft topological space $(f, \tilde{\tau})$. Then, the following are equivalent:

i. $g \in \tilde{\tau}$

ii. $\tilde{\tau}_{g} \tilde{\subseteq} \tilde{\tau}$.

Proof: $(i) \Rightarrow(i i)$ : Let $g \in \tilde{\tau}$. Take as given $\forall h \in \tilde{\tau}_{g}$. From the defi of soft subspace topology, $h=m \tilde{\cap} g$, where $\exists m \in \tilde{\tau}$. Since $g \in \tilde{\tau}$ and $m \in \tilde{\tau}$ then, $h \in \tilde{\tau}$. Hence $\tilde{\tau}_{g} \tilde{\subseteq} \tilde{\tau}$.

(ii) $\Rightarrow(i)$ : Assume that $\tilde{\tau}_{g} \tilde{\tilde{\tau}} \tilde{\tau}$. Since $g \in \tilde{\tau}_{g}$ then, $g \in \tilde{\tau}$.

\section{SOFT DITOPOLOGICAL SPACES}

In this section, the concept of soft ditopological (SDT) space on a soft set with two structures on it is introduced - a soft topology and a soft subspace topology. The first one is used to describe soft openness properties of a soft topological space while the second one deals with its sub - soft openness properties. This structure enables to study with all soft open sets can be obtained on a soft set.

\subsection{Definition of Soft Ditopological Spaces}

In this section study of soft ditopological spaces is initiated. Soft ditopological space on a soft set is introduced and definitions of soft open set, soft closed set, soft closure, soft interior and soft neighborhood in a soft ditopological space are given. Among the main properties of them, some important relations between soft interior and soft closure operations have been investigated. The comparison of soft ditopological spaces is defined. A constructive proof of every soft ditopological space of a soft set is a soft topological space over the soft set and a soft topological space is not need to be soft ditopological space is provided. All theorems characterizing behaviour of SDT and give suitable examples are proved.

DEFINITION 14. Let $f$ be a nonempty soft set over the universe $U, g \tilde{\subseteq} f, \tilde{\tau}$ be a soft topology on $f$ and $\tilde{\tau}_{g}$ be a soft subspace topology on $g$. Then, $\left(f, \tilde{\tau}, \tilde{\tau}_{g}\right)$ is called a soft ditopological space which is abbreviated as SDT-space.

A pair $\tilde{\delta}=\left(\tilde{\tau}, \tilde{\tau}_{g}\right)$ is called a soft ditopology over $f$ and the members of $\tilde{\delta}$ are said to be $\tilde{\delta}$-soft open in $f$.

The complement of $\tilde{\delta}$-soft open set is called $\tilde{\delta}$-soft closed.
EXAMPLE 5. Let us consider all soft subsets on $f$ in the exam 1 Let $\tilde{\tau}=\left\{\Phi, f, f_{2}, f_{11}, f_{13}\right\}$ be a soft topology on $f$. If $g=f_{9}$, then $\tilde{\tau}_{g}=\left\{\Phi, f_{5}, f_{7}, f_{9}\right\}$, and $\left(g, \tilde{\tau}_{g}\right)$ is a soft topological subspace of $(f, \tilde{\tau})$.

Hence, we get soft ditopology over $f$ as $\tilde{\delta}=$ $\left\{\Phi, f, f_{2}, f_{5}, f_{7}, f_{9}, f_{11}, f_{13}\right\}$.

THEOREM 15. Every soft ditopological space of a soft set is a soft topological space over the soft set.

Proof: Let $\tilde{\delta}=\left(\tilde{\tau}, \tilde{\tau}_{g}\right)$ be a soft ditopology over $f$. Then,

i. $\Phi, f \tilde{\in} \tilde{\delta}$

ii. Since $h_{i} \tilde{\subseteq} f$ then, $h_{i} \tilde{\in} \tilde{\delta}$, for all $i \in I$. Moreover, $\tilde{\bigcup}_{i \in I} h_{i} \tilde{\subseteq} f$ it follows that, $\tilde{\bigcup}_{i \in I} h_{i} \tilde{\in} \tilde{\delta}$. On the other hand,

$$
\tilde{\bigcup}_{i \in I}\left(h_{i} \tilde{\cap} g\right)=\left(\tilde{\bigcup}_{i \in I} h_{i}\right) \tilde{n} g
$$

for $\tilde{\tau}=\left\{h_{i} \tilde{\subseteq} f: i \in I\right\}$.

Thus, the union of any number of soft subsets in $\tilde{\delta}$ belongs to $\tilde{\delta}$.

iii. Since $h_{i} \tilde{\subseteq} f$, for all $i \in I^{\prime}, I^{\prime}$ finite soft set, so that $\tilde{\bigcup}_{i \in I^{\prime}} h_{i} \tilde{\subseteq} f$. Thus $\tilde{U}_{i \in I^{\prime}} h_{i} \tilde{\in} \tilde{\delta}$. Moreover,

$$
\tilde{\bigcap}_{i=1}^{n}\left(h_{i} \tilde{\cap} g\right)=\left(\tilde{\bigcap}_{i=1}^{n} h_{i}\right) \tilde{n} g
$$

Thus, the finite intersection of soft sets in $\tilde{\delta}$ belongs to $\tilde{\delta}$. Hence $\tilde{\delta}$ is a soft topology over $f$.

Although every soft ditopological space of a soft set is a soft topological space over the soft set, a soft topological space is not need to be soft ditopological space. The following example shows that:

EXAMPLE 6. Consider the topology $\tilde{\tau}$ and $\tilde{\tau}_{g}$ are defined in exam 5. Then, $(f, \tilde{\tau}) \neq(f, \tilde{\delta})$. Refer the theorem 13 if $g \in \tilde{\tau}$ then, $(f, \tilde{\tau})=\left(f, \tilde{\tau}, \tilde{\tau}_{g}\right)$.

Definition 16. Let $\left\{\tilde{\delta}_{i}\right\}_{i \in I}=\left(\left\{\tilde{\tau}_{i}\right\}_{i \in I},\left\{\tilde{\tau}_{g}\right\}_{i \in I}\right)$ be the family of all soft ditopologies on $f$. Then, $\tilde{\delta}$ - soft coarser (or weaker) denoted by $\tilde{\delta}_{i} \leq \tilde{\delta}_{j}$, is defined by

$$
\left(\tilde{\delta}_{i} \leq \tilde{\delta}_{j}\right) \Leftrightarrow\left(\tilde{\tau}_{i} \tilde{\subseteq} \tilde{\tau}_{j}, \tilde{\tau_{g_{i}}} \tilde{\subseteq} \tilde{\tau_{g_{j}}}\right)
$$

In this case $\tilde{\delta}_{i}$ is said to be soft coarser (or weaker) than $\tilde{\delta}_{j}$. If either $\tilde{\delta}_{i} \leq \tilde{\delta}_{j}$ or $\tilde{\delta}_{j} \leq \tilde{\delta}_{i}$, then $\tilde{\delta}_{i}$ is soft comparable with $\tilde{\delta}_{j}$.

EXAmple 7. Let $U=\left\{u_{1}, u_{2}, u_{3}\right\}$ and $E=\left\{e_{1}, e_{2}\right\} . f \in S$ and

$$
f=\left\{\left(e_{1},\left\{u_{1}, u_{2}, u_{3}\right\}\right),\left(e_{2},\left\{u_{2}\right\}\right)\right\}
$$

Then,

$$
\tilde{\tau}_{1}=\left\{\Phi, f, f_{1}, f_{4}\right\}
$$

and

$$
\tilde{\tau}_{2}=\left\{\Phi, f, f_{1}, f_{2}, f_{4}, f_{8}, f_{9}, f_{12}\right\}
$$

are soft topologies on $f$ where $f_{1}, f_{2}, f_{3}, f_{4}, f_{5}, f_{6}, f_{7}, f_{8}, f_{9}, f_{10}, f_{11}, f_{12}, f_{13}, f_{14}, f_{15}, f_{16}$ are soft sets over $f$, defined as follows: 


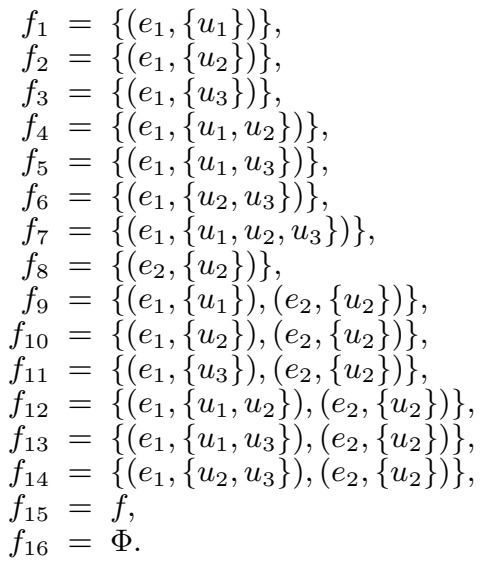

If $g=f_{1}$, then $\tilde{\tau_{g_{1}}}=\left\{\Phi, f_{1}\right\}$ and $\tilde{\tau_{g_{2}}}=\left\{\Phi, f_{1}\right\}$. Then, $\left(g, \tilde{\tau_{g_{1}}}\right)$ and $\left(g, \tilde{\tau}_{g_{2}}\right)$ are soft topological subspaces of $(f, \tilde{\tau})$.

Hence, we get soft ditopologies $\tilde{\delta}_{1}$ and $\tilde{\delta}_{2}$ over $f$ as,

$$
\tilde{\delta}_{1}=\left\{\Phi, f, f_{1}, f_{4}\right\}
$$

and

$$
\tilde{\delta}_{2}=\left\{\Phi, f, f_{1}, f_{2}, f_{4}, f_{8}, f_{9}, f_{12}\right\}
$$

Here, $\tilde{\tau}_{1} \tilde{\subseteq} \tilde{\tau}_{2}$ and $\tilde{\tau_{g_{1}}} \tilde{\subseteq} \tilde{\tau}_{g_{2}}$. Then, $\tilde{\delta}_{1} \leq \tilde{\delta}_{2}$. So, $\tilde{\delta}_{1}$ is soft coarser (or weaker) than $\tilde{\delta}_{2}$.

Definition 17. Let $h \tilde{\subseteq} f$. Then, $\tilde{\delta}$-interior of $h$, denoted by $(g)_{\tilde{\delta}}^{\circ}$, is defined by

$$
(h)_{\tilde{\delta}}^{\circ}=\tilde{\bigcup}\{h: k \tilde{\subset} h, k \text { is } \tilde{\delta} \text {-soft open }\}
$$

The $\tilde{\delta}$ - closure of $h$, denoted by $(\bar{h})_{\tilde{\delta}}$, is defined by

$$
(\bar{h})_{\tilde{\delta}}=\tilde{\bigcap}\{k: h \tilde{\subset} k, k \text { is } \tilde{\delta} \text {-soft closed }\}
$$

Note that $(h)_{\tilde{\delta}}^{\circ}$ is the biggest $\tilde{\delta}$-soft open set that contained in $h$ and $(\bar{h})_{\tilde{\delta}}$ is the smallest $\tilde{\delta}$-soft closed set that containing $h$.

THEOREM 18. Let $\left(f, \tilde{\tau}, \tilde{\tau}_{g}\right)$ be a SDT-space and $h \tilde{\subseteq} f$. Then, $h$ is a $\tilde{\delta}$-soft open set if and only if $h=(h)_{\tilde{\delta}}^{\circ}$.

Proof: If $h$ is $\tilde{\delta}$-soft open set then, $h=(h)_{\tilde{\delta}}^{\circ}$ where $h$ is the biggest $\tilde{\delta}$-soft open set that contained by $h$. Conversely, it is known that $(h)_{\tilde{\delta}}^{\circ}$ is $\tilde{\delta}$-soft open set, and if $h=(h)_{\tilde{\delta}}^{\circ}$, then $h$ is $\tilde{\delta}$-soft open set.

THEOREM 19. Let $\left(f, \tilde{\tau}, \tilde{\tau}_{g}\right)$ be a SDT-space and $h \tilde{\subseteq} f$. Then, $h$ is a $\tilde{\delta}$-closed soft set if and only if $h=(\bar{h})_{\tilde{\delta}}$.

Proof: The proof is trivial.

THEOREM 20. Let $\left(f, \tilde{\tau}, \tilde{\tau}_{g}\right)$ be a SDT-space and $h \tilde{\subseteq} f$. Then, $(h)_{\tilde{\delta}}^{\circ} \tilde{\subseteq} h \tilde{\subseteq}(\bar{h})_{\tilde{\delta}}$.

Proof: By the defi of $\tilde{\delta}$ - interior of $h$,

$(h)_{\tilde{\delta}}^{\circ}=\tilde{\bigcup}\left\{k_{i}: h_{i} \in \tilde{\delta}, k_{i} \tilde{\subseteq} h, i \in I\right\}$. Then, $k_{i} \tilde{\subseteq} h$ and $\bigcup_{i \in I} k_{i} \tilde{\subseteq} h$ for all $i \in I$. So, $(h)_{\tilde{\delta}}^{\circ} \tilde{\subseteq} h$.

If we consider the defi of $\tilde{\delta}$ - closure of $h$, we obtain that, $(\bar{h})_{\tilde{\delta}}=\tilde{\bigcup}\left\{h_{i}: h_{i}^{c} \in \tilde{\delta}, h \tilde{\subseteq} h_{i}, i \in I\right\}$. Then, $h \tilde{\subseteq} h_{i}$ and $h \tilde{\subseteq} \tilde{\bigcap}_{i \in I} h_{i}$ for all $i \in I$. So, $h \tilde{\subseteq}(\bar{h})_{\tilde{\delta}}$. Hence we get

$$
(h)_{\tilde{\delta}}^{\circ} \tilde{\subseteq} h \tilde{\subseteq}(\bar{h})_{\tilde{\delta}}
$$

DEFINITION 21. Let $\left(f, \tilde{\tau}, \tilde{\tau}_{g}\right)$ be a SDT-space and $\tilde{\mathcal{B}}_{\tilde{\delta}} \tilde{\subseteq} \tilde{\delta}$. If every element of $\tilde{\delta}$ can be written as a union of element of $\tilde{\mathcal{B}}_{\tilde{\delta}}$, then $\tilde{\mathcal{B}}_{\tilde{\delta}}$ is called $\tilde{\delta}$-soft basis for $\left(f, \tilde{\tau}, \tilde{\tau}_{g}\right)$. Each element of $\tilde{\mathcal{B}}_{\tilde{\delta}}$ is called soft ditopological basis element.

We can shortly define it like that,

$$
\tilde{\mathcal{B}}_{\tilde{\delta}} \tilde{\subseteq} \tilde{\delta}, \tilde{\delta} \text {-soft basis } \Leftrightarrow(\forall h \in \tilde{\delta})\left(\exists \Psi \tilde{\subset} \tilde{\mathcal{B}}_{\tilde{\delta}}\right):\left(h=\tilde{\bigcup}_{k \in \Psi} k\right)
$$

EXAmple 8. Considering exam 1 and exam $3, \tilde{\mathcal{B}}_{\tilde{\delta}}=$ $\left\{\Phi, f_{1}, f_{2}, f_{4}, f_{5}\right\}$ is a soft basis for $\left(f, \tau, \tilde{\tau}_{g}\right)$.

THEOREM 22. Let $\tilde{\mathcal{B}}_{\tilde{\delta}}$ and $\tilde{\mathcal{B}}_{\tilde{\delta}}^{\prime}$ be SDT-bases for $\left(f, \tilde{\tau}, \tilde{\tau}_{g}\right)$ and $\left(f, \tilde{\tau}^{\prime}, \tilde{\tau}_{g}^{\prime}\right)$, respectively. If $\tilde{\mathcal{B}}_{\tilde{\delta}}^{\prime} \tilde{\subseteq} \tilde{\mathcal{B}}_{\tilde{\delta}}$, then $\left(f, \tilde{\tau}^{\prime}, \tilde{\tau}_{g}^{\prime}\right) \tilde{\subseteq}\left(f, \tilde{\tau}, \tilde{\tau}_{g}\right)$.

Proof: Let $\tilde{\mathcal{B}}_{\tilde{\delta}}^{\prime} \tilde{\subseteq} \tilde{\mathcal{B}}_{\tilde{\delta}}$. Then for each $h \in \tilde{\delta}$ and $k \in \tilde{\mathcal{B}}_{\tilde{\delta}}^{\prime}$,

$$
h=\tilde{\bigcup}_{k \in \tilde{\mathcal{B}}_{\tilde{\delta}}^{\prime}} k=\tilde{\bigcup}_{k \in \tilde{\mathcal{B}}_{\tilde{\delta}}} k
$$

Therefore $h \in \tilde{\delta}$, hence $\left(f, \tilde{\tau}^{\prime}, \tilde{\tau}_{g}^{\prime}\right) \tilde{\subseteq}\left(f, \tilde{\tau}, \tilde{\tau}_{g}\right)$.

DEFINITION 23. Let $\left(f, \tilde{\tau}, \tilde{\tau}_{g}\right)$ be a SDT-space and $\left(e_{i_{f}}\right)_{j} \tilde{\in} f$. If there is a $\tilde{\delta}$-soft open set $h$ such that $\left(e_{i_{f}}\right)_{j} \tilde{\in} h$, then $h$ is called $\tilde{\delta}$-soft open neighborhood (or $\tilde{\delta}$-soft neighborhood) of $\left(e_{i_{f}}\right)_{j}$. The set of all $\tilde{\delta}$-soft neighborhood of $\left(e_{i_{f}}\right)_{j}$, denoted $\tilde{\mathcal{V}}_{\tilde{\delta}}\left(\left(e_{i_{f}}\right)_{j}\right)$, is called family of $\tilde{\delta}$-soft neighborhood of $\left(e_{i_{f}}\right)_{j}$, that is

$$
\tilde{\mathcal{V}}_{\tilde{\delta}}\left(\left(e_{i_{f}}\right)_{j}\right)=\left\{h: h \tilde{\in} \tilde{\delta},\left(e_{i_{f}}\right)_{j} \tilde{\in} h\right\}
$$

\subsection{Separation Axioms in Soft Ditopological Spaces}

Separation axioms for a soft ditopological space $\left(f, \tilde{\tau}, \tilde{\tau}_{g}\right)$ are introduced by requesting corresponding separation properties for its soft topology $\tilde{\tau}$ and soft subspace topology $\tilde{\tau}_{g}$.

DEFINITION 24. Let $\left(f, \tilde{\tau}, \tilde{\tau}_{g}\right)$ be a SDT-space over $f$ and $\left(e_{i_{f}}\right)_{1},\left(e_{i_{f}}\right)_{2} \tilde{\in} f$ be such that $\left(e_{i_{f}}\right)_{1} \neq\left(e_{i_{f}}\right)_{2}$. If there exist $a \tilde{\tau}$ open set $v_{1}$ and $\tilde{\tau}_{g}$ open set $v_{2}$ such that " $\left(e_{i_{f}}\right)_{1} \tilde{\in} v_{1}$ and $\left(e_{i_{f}}\right)_{2} \notin v_{1}$ " or " $\left(e_{i_{f}}\right)_{2} \tilde{\in} v_{2}$ and $\left(e_{i_{f}}\right)_{1} \notin v_{2}$ " then, $\left(f, \tilde{\tau}, \tilde{\tau}_{g}\right)$ is called a soft di-T $T_{0}$ space and denoted by $\tilde{T}_{0} \tilde{\delta}$.

DEFINITION 25. Let $\left(f, \tilde{\tau}, \tilde{\tau}_{g}\right)$ be a SDT-space over $f$ and $\left(e_{i_{f}}\right)_{1},\left(e_{i_{f}}\right)_{2} \tilde{\in} f$ be such that $\left(e_{i_{f}}\right)_{1} \neq\left(e_{i_{f}}\right)_{2}$. If there exist a $\tilde{\tau}$ open set $v_{1}$ and $\tilde{\tau}_{g}$ open set $v_{2}$ such that " $\left(e_{i_{f}}\right)_{1} \tilde{\in} v_{1}$ and $\left(e_{i_{f}}\right)_{2} \notin v_{1}$ " and " $\left(e_{i_{f}}\right)_{2} \tilde{\in} v_{2}$ and $\left(e_{i_{f}}\right)_{1} \notin v_{2}$ " then, $\left(f, \tilde{\tau}, \tilde{\tau}_{g}\right)$ is called a soft di-T $T_{1}$ space and denoted by $\tilde{T}_{1}$.

DEFINITION 26. Let $\left(f, \tilde{\tau}, \tilde{\tau}_{g}\right)$ be a SDT-space. If for each pair of distinct soft points $\left(e_{i_{f}}\right)_{1},\left(e_{i_{f}}\right)_{2} \tilde{\in} f$ there exist a $\tilde{\tau}$ open set $v_{1}$ and $\tilde{\tau}_{g}$ open set $v_{2}$ such that $\left(e_{i_{f}}\right)_{1} \tilde{\in} v_{1},\left(e_{i_{f}}\right)_{2} \tilde{\in} v_{2}$ and $v_{1} \tilde{\cap} v_{2}=\Phi$ then $\left(f, \tilde{\tau}, \tilde{\tau}_{g}\right)$ is called a SDT- Hausdorff space. SDT-Hausdorff space is named as soft di-T $T_{2}$ space and denoted by $\tilde{T}_{2} \tilde{\delta}$ 
EXAMPLE 9. Let $U=\left\{u_{1}, u_{2}, u_{3}\right\}$ and $E=\left\{e_{1}, e_{2}\right\} . f \in S$ and

$$
f=\left\{\left(e_{1},\left\{u_{1}, u_{2}, u_{3}\right\}\right),\left(e_{2},\left\{u_{2}, u_{3}\right\}\right)\right\}
$$

Then,

$$
\tilde{\tau}=\left\{\Phi, f, f_{1}, f_{2}, f_{3}, f_{4}, f_{5}, f_{6}, f_{7}, f_{8}, f_{9}, f_{10}, f_{11}, f_{12}, f_{13}, f_{14}\right\}
$$

is a soft topology on $f$ where $f_{1}, f_{2}, f_{3}, f_{4}, f_{5}, f_{6}, f_{7}, f_{8}, f_{9}, f_{10}, f_{11}, f_{12}, f_{13}, f_{14}$ are soft sets over $f$, defined as follows:

$$
\begin{aligned}
f_{1} & =\left\{\left(e_{1},\left\{u_{1}\right\}\right)\right\}, \\
f_{2} & =\left\{\left(e_{1},\left\{u_{2}\right\}\right)\right\}, \\
f_{3} & =\left\{\left(e_{1},\left\{u_{1}, u_{2}\right\}\right)\right\}, \\
f_{4} & =\left\{\left(e_{2},\left\{u_{2}\right\}\right)\right\}, \\
f_{5} & =\left\{\left(e_{2},\left\{u_{3}\right\}\right)\right\}, \\
f_{6} & =\left\{\left(e_{2},\left\{u_{2}, u_{3}\right\}\right)\right\}, \\
f_{7} & =\left\{\left(e_{1},\left\{u_{1}\right\}\right),\left(e_{2},\left\{u_{2}\right\}\right)\right\}, \\
f_{8} & =\left\{\left(e_{1},\left\{u_{1}\right\}\right),\left(e_{2},\left\{u_{3}\right\}\right)\right\}, \\
f_{9} & =\left\{\left(e_{1},\left\{u_{1}\right\}\right),\left(e_{2},\left\{u_{2}, u_{3}\right\}\right)\right\}, \\
f_{10} & =\left\{\left(e_{1},\left\{u_{2}\right\}\right),\left(e_{2},\left\{u_{2}\right\}\right)\right\}, \\
f_{11} & =\left\{\left(e_{1},\left\{u_{2}\right\}\right),\left(e_{2},\left\{u_{3}\right\}\right)\right\}, \\
f_{12} & =\left\{\left(e_{1},\left\{u_{2}\right\}\right),\left(e_{2},\left\{u_{2}, u_{3}\right\}\right)\right\}, \\
f_{13} & =\left\{\left(e_{1},\left\{u_{1}, u_{2}\right\}\right),\left(e_{2},\left\{u_{2}\right\}\right)\right\}, \\
f_{14} & =\left\{\left(e_{1},\left\{u_{1}, u_{2}\right\}\right),\left(e_{2},\left\{u_{3}\right\}\right)\right\} .
\end{aligned}
$$

If $g=f_{10}$, then $\tilde{\tau}_{g}=\left\{\Phi, f_{2}, f_{4}, f_{10}\right\}$ and $\left(g, \tilde{\tau}_{g}\right)$ is a soft topological subspace of $(f, \tilde{\tau})$.

Hence, we get soft ditopology $\tilde{\delta}$ over $f$,

$\tilde{\delta}=\left\{\Phi, f, f_{1}, f_{2}, f_{3}, f_{4}, f_{5}, f_{6}, f_{7}, f_{8}, f_{9}, f_{10}, f_{11}, f_{12}, f_{13}, f_{14}\right\}$

For distinct soft points $\left(e_{1_{f}}\right)_{1}=\left\{\left(e_{1},\left\{u_{1}\right\}\right)\right\} \tilde{\in} f$ and $\left(e_{1_{f}}\right)_{2}=$ $\left\{\left(e_{1},\left\{u_{2}\right\}\right)\right\} \tilde{\in} f$,

$f_{1} \tilde{\in} \tilde{\tau}$ and $f_{2} \tilde{\in} \tilde{\tau}_{g}$ such that,

$$
\left(e_{1_{f}}\right)_{1} \tilde{\in} f_{1},\left(e_{1_{f}}\right)_{2} \tilde{\in} f_{2} \text { and } f_{1} \tilde{\cap} f_{2}=\Phi .
$$

For distinct soft points $\left(e_{2_{f}}\right)_{1}=\left\{\left(e_{2},\left\{u_{2}\right\}\right)\right\} \tilde{\in} f$ and $\left(e_{2_{f}}\right)_{2}=$ $\left\{\left(e_{2},\left\{u_{3}\right\}\right)\right\} \tilde{\in} f$,

$f_{4} \tilde{\in} \tilde{\tau}_{g}$ and $f_{5} \tilde{\in} \tilde{\tau}$ such that,

$$
\left(e_{2_{f}}\right)_{1} \tilde{\in} f_{4},\left(e_{2_{f}}\right)_{2} \tilde{\in} f_{5} \text { and } f_{4} \tilde{\cap} f_{5}=\Phi .
$$

Hence, $\left(f, \tilde{\tau}, \tilde{\tau}_{g}\right)$ is a SDT- Hausdorff space over $f$.

THEOREM 27. Every finite point $\tilde{\delta}$-soft set in a SDT-Hausdorff space is $\tilde{\delta}$-soft closed set.

Proof: $\left(f, \tilde{\tau}, \tilde{\tau}_{g}\right)$ be a SDT-Hausdorff space. It suffices to show that every one soft point set $\left\{\left(e_{i_{f}}\right)_{j}\right\}$ is $\tilde{\delta}$-soft closed. If $\left(e_{i_{f}}\right)_{2} \tilde{\in} f$ and $\left(e_{i_{f}}\right)_{2} \neq\left(e_{i_{f}}\right)_{1}$, then $\left(e_{i_{f}}\right)_{1}$ and $\left(e_{i_{f}}\right)_{2}$ have disjoint $\tilde{\delta}$ soft neighborhoods $h_{1}$ and $h_{2}$ respectively. Since $h_{1}$ does not soft intersect $\left\{\left(e_{i_{f}}\right)_{2}\right\}$, the point $\left(e_{i_{f}}\right)_{1}$ cannot belong to the $\tilde{\delta}$-soft closure of the set $\left\{\left(e_{i_{f}}\right)_{2}\right\}$. As a result, $\left\{\overline{\left(e_{i_{f}}\right)_{1}}\right\}_{\tilde{\delta}}=\left\{\left(e_{i_{f}}\right)_{1}\right\}$ itself, hence it is $\tilde{\delta}$-soft closed.

\section{CONCLUSION}

In this work, soft ditopological space on a soft set was defined and its related properties and separation axioms were studied. Then, the relation between soft topology and soft ditopology was presented.
It considers some of the new results and consequences, which could be useful from the point of view of soft set theory, that were not studied at all. Based on this with new subspace theory, some further work can be done on the properties of soft ditopological spaces, which may be useful to characterize some other soft mathematical concepts and structures.

\section{REFERENCES}

[1] Ali, M.I., Feng, F., Liu, X., Min, W.K., Shabir, M., On some new operations in soft set theory, Computers and Mathematics with Applications 57, 1547-1553, 2009.

[2] Aygünoglu, A. Aygün, H., Some notes on soft topological spaces, Neural Computation and Application, 2011.

[3] Aktaş, H. ve Çağman, N., Soft sets and soft groups, Information Sciences, 177(1), 2726-2735, 2007.

[4] Çağman, N., Enginoğlu, S., Soft set theory and uni-int decision making, European Journal of Operational Research 207, 848-855, 2010

[5] Dizman, T. S., Sostakb, A., Yuksel, S., Soft Ditopological Spaces, Filomat 30:1 (2016), 209 - 222.

[6] Ittanagi, Basavaraj M., Soft Bitopological Spaces, International Journal of Computer Applications (0975 8887), Volume 107 - No. 7, December 2014.

[7] Maand, X., Zhan, J., Characterizations of three kinds of hemirings by fuzzy soft h-ideals, J Intell Fuzzy Syst 24(3), 535-548, 2013.

[8] Maji, P.K., Biswas, R., Roy, A.R., An Application of Soft Sets in A Decision Making Problem, Computers and Mathematics with Applications 44, 1077-1083, 2002.

[9] Maji, P.K., Biswas, R., Roy, A.R., Soft set theory, Computers and Mathematics with Applications 45, 555-562, 2003.

[10] Molodtsov, D.A., Soft set theory-first results, Computers and Mathematics with Applications 37, 19-31, 1999.

[11] Şenel, G., Çağman, N., Soft Topological Subspaces, Annals of Fuzzy Mathematics and Informatics, vol.10, no : 4, 525 535, 2015.

[12] Shabir, M., Naz, M., On soft topological spaces, Computers and Mathematics with Applications 61, 1786-1799, 2011. 